\title{
Who goes where from here?
}

This survey of the present state of neurosciences is necessarily a kind of snapshot, one taken from an idiosyncratic point of view. Others might well have paid more attention to other aspects of the functioning of typical nervous systems - the biochemistry of the brain, the psychological tests that can be made of the functioning of these systems and insight that may be gleaned from the way in which typical nervous systems respond to injury, either accidental or deliberate (as in surgery). Much the same conclusion would no doubt emerge from a survey of some other field - high-energy physics for example. The most vivid impressions are those conveyed most forcefully by those accidentally encountered. Nature is grateful to those who have been good enough patiently to answer the questions importunately put to them.

A few generalizations are appropriate. First, the present ferment in the neurosciences is not new. What has happened in the past few years is merely that the community of the neuroscientists has grown, perhaps by an order of magnitude in ten years. Second, the kinds of problems with which people now contend are not very different from those of the 1950s, or for that matter of the 1930s. As always, the most interesting problems are those which cannot unambiguously be resolved for lack of data.

Third, however, a good deal of water has now run under the bridge. Several features of the typical nervous system and even of the human brain are well established. The chances are good that the cooperative functioning of aggregations of neurones will indeed account for the way in which brains of all degrees of complexity perform. One point that needs urgently to be established is whether the behaviour of such a cooperative aggregate will indeed be sharper, less fuzzy at the edges, than the behaviour of single neurones. That must surely be a challenge for the physicists and those in artificial intelligence.

There is also a need for a better understanding of the metabolism of neuronal cells. Some specific interactions between those materials unambiguously known to be neurotransmitters and the downstream cells are reasonably well understood. But by what mechanisms is the replenishment of neuronal transmitters in the presynaptic cell regulated? And what balance can be struck between the effects of neurotransmitters at synaptic junctions and the more generalized effects of neuroactive materials in the intercellular medium? Some indication of the importance of these metabolic questions may be gleaned from the fact that no less than 14 per cent of the energy consumption of the adult human being is expended on the metabolism of the human brain.

Other issues arise in the anatomical description of real nervous systems. The general tendency is for there to be a more or less geometrical representation of the outside world on the surface of the cortex, in the visual and in other sensory systems, but there is at present only the sketchiest understanding of how such representations may be interconnected. Practically, however, these interconnections are crucial to proper functioning. One can avoid a visible obstacle in one's path without thinking very hard. Is this a field in which psychology may more rapidly contribute to understanding than plain painstaking anatomy?

The conceptual part of the problem of neurobiology also deserves more attention than it has received. Grand philosophical questions - what is consciousness, and so on, are probably less important than more strictly operational questions - by what means do people learn to assign names to familiar objects, providing themselves with a kind of lexicon for the images formed in the visual cortex? Is Chomsky's view that the structure of language implies a kind of universal grammar reflected in the anatomy of the regions of the brain and, if so, what kinds of connections are implied?

The growth of the American Society for Neuroscience in the past few years is at once a measure of the enthusiasm of people (mostly relatively young) for this absorbing field of enquiry and of the willingness of the grant-making agencies to back that enthusiasm with grants. But the growth cannot continue indefinitely. The society's boast, in recent years, that by the end of the century there will be more neuroscientists than adult citizens in the United States is a poor joke. Has the time come when the neurosciences should work out a strategy for maturity? 